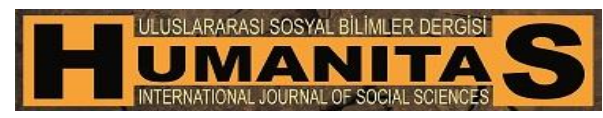

$\begin{array}{ll}\text { Humanitas, 2017; 5(10): 333-361 } & \text { http://humanitas.nku.edu.tr } \\ \text { ISSN: } 2147-088 X & \text { DOI: } 10.20304 / \text { humanitas. } 342072\end{array}$

Araştırma-İnceleme

\title{
ACQUISITION DU SUBJONCTIF PAR LES ÉTUDIANTS TURCS $^{1}$
}

\section{Yusuf TOPALOĞLU ${ }^{2}$}

Résumé : La présente étude traite le sujet de la dimension linguistique des enjeux dans le contexte du subjonctif que les étudiants universitaires turcs rencontrent au cours de leur apprentissage du français. L'acquisition et le transfert du mode sont des principaux problèmes rencontrés. En effet, nous avons expérimenté nous-mêmes ces difficultés d'acquisition du subjonctif de très près, comme étudiants dans le passé et pédagogue d'aujourd'hui. Nous croyons que le principal facteur sous-jacent de cette difficulté est la différence entre les langues. La présente étude a été étudiée dans le cadre de la linguistique appliquée. D'ailleurs, le subjonctif et les modalités radicales proposées pour être son équivalent ont été examinés avec une approche contrastive. L'idée que le mode subjonctif comme morphologique et fonctionnelle est très différent, pour les étudiants universitaires turcs, est mis en avant et les différences dues à des difficultés dans le processus d'acquisition sont étudiés. Cette étude a été évaluée dans le cadre de méthode quantitative. Un questionnaire technique a été utilisé dans la collecte des données quantitatives qui ont été analysées par la suite. Le questionnaire se compose de 37 questions. D'après les données obtenues, les similitudes facilitent l'acquisition et les différences la rendent difficile; le subjonctif ne possède pas complètement un caractère de xénité, ne constitue pas non plus un élément commun pour les étudiants universitaire turcs.

Mots-clés: Linguistique Appliquée, Linguistique Contrastive, Acquisition du Langage, Apprentissage du Langage, Subjonctif.

\section{TÜRK ÖĞRENCILLERIN FRANSIZCA ÖZNELLIKK KIPİ EDINIMİ}

Öz : $\mathrm{Bu}$ çalışma, Türk öğrencilerin Fransızca öğrenim sürecinde karşılaştıkları zorlukların dilsel boyutunun öznellik kipi bağlamında karşıtsal bir yaklaşımla incelenmesini konu edinmektedir. Söz konusu

\footnotetext{
${ }^{1}$ Cet article est basé sur la thèse intutilée Türk Üniversite Öğrencilerinin Fransizca Öznellik Kipi Edinimi, soutenue à l'université de Çukorova, le 9 Décembre 2016, laquelle a bénéficié d'un soutien de TÜBİTAK dans le cadre d'une bourse de recherche doctorale (numéro de projet : 1059B141400364).

${ }^{2}$ Okt. Dr., Namık Kemal Üniversitesi, Yabancı Diller Yüksekokulu. ytopaloglu@nku.edu.tr
} 
kipin hem öğrenimi hem aktarımı karşılaşılan en önemli sorunlardan biridir. Gerçekten, Fransızca öznellik kipi öğreniminin gerek öğrenciler gerekse eğitimciler için güçlük çekilen konuların başındadır. Bu zorluğun temelinde yatan etkenin ise, diller arasındaki farklılıklardan kaynaklanıyor olduğunu düşünmekteyiz. Uygulamalı dilbilimin karşıtsal dilbilim alanına giren bu çalışmada, Fransızca öznellik kipi ile Türkçede eşdeğeri olan tasarlama kipleri karşıtsal bir yaklaşımla incelenmektedir. Öznellik kipinin biçimsel ve işlevsel olarak Türk üniversite öğrencilerine farklı olduğu görüşü ileri sürülmekte ve farklılıkların, öğrenim sürecinde sebep olduğu zorluklar araştırılmaktadır. $\mathrm{Bu}$ çalışma, nicel araştırma tekniği çerçevesinde değerlendirilmiştir. Veri toplamada, sormaca tekniği kullanılmış, sayısal veriler elde edilmiş ve analizleri yapılmıştır. Söz konusu sormaca, 37 sorudan oluşturulmuştur. Elde edilen verilere göre, dilsel benzerlikler öğrenimi kolaylaştırmakta, farklılıklar ise, zorlaştırmaktadır; Türk üniversite öğrencileri için öznellik kipinin, tamamen yabancıl öğe (fr. Xénité) özelliği taşımadığı gibi tamamen aynılık özelliği de taşımadığı anlaşılmıştır.

Anahtar Sözcükler: Uygulamalı Dilbilim, Karşıtsal Dilbilim, Dil Edinimi, Dil Öğretimi, Öznellik Kipi.

\section{Introduction}

Chaque individu de toute communauté linguistique recourt en générale à la langue acquise naturellement dans un contexte familial et communautaire pour établir la communication. Avec cette première langue acquise, appelée communément langue maternelle, l'individu exprime ses pensées, sentiments et craintes (Korkmaz, 1992, p. 8). Après avoir entendu et appris la langue maternelle, on commence à apprendre des langues différentes. Chacune de ces langues qui sont apprises en dehors de la langue maternelle est une langue étrangère. Chaque langue acquise précédemment, qu'elle soit langue maternelle ou langue étrangère, a une influence majeure sur l'apprentissage des langues par la suite. En effet, chaque apprenant se réfère à ces langues en question au cours de l'acquisition d'une nouvelle langue. Cette référence peut entrainer des effets aussi bien positifs que négatifs sur l'apprentissage de la langue, qui sont catégorisés selon plusieurs critères (Lado, 1957 ; Bouton, 1984 ; Porcher, 2003 ; Gaonac'h, 2009). Le plus important d'entre eux est le phénomène du transfert. D'après Corder (1981, p. 99-100), le transfert est la continuité du comportement préexistant. En effet, la structure de la langue maternelle a tendance à être transférée dans la langue cible. L'apprenant pourrait transférer les paramètres, les formes de phrase, les variations, les chiffres et les genres de sa langue maternelle. En conséquence, le transfert peut être défini comme un phénomène qui rend facile ou difficile l'apprentissage d'une langue étrangère en amenant les habitudes linguistiques dans une autre langue. Il existe deux types de transfert : Le transfert positif et le transfert négatif. Quand il s'agit de la similitude lorsqu'on transfère d'une langue à l'autre, l'apprentissage est facile, on parle alors du transfert positif ; quand il y a une différence, l'apprentissage est difficile, il s'agirait du transfert négatif (Březinová, 2009, p. 7). Quant aux 
transferts négatifs résultant des langues en question, ils donnent lieu aux interférences entre les deux langues. L'interférence est un phénomène qui peut se produire par le biais de linteraction des langues. Les interférences sont souvent basées sur les transferts négatifs. Celles-ci peuvent être définies comme les erreurs qui se révèlent lors du transfert. À cause de ces interférences, les étudiants qui apprennent une langue étrangère commettent des fautes et des erreurs de manières très différentes. Ces fautes et erreurs en question rendent difficile l'apprentissage d'une langue étrangère. La faute est liée à la performance ; il s'agit de ne pas suivre les règles de façon consciente. Quant à l'erreur, elle est liée à la compétence; il s'agit des erreurs commises inconsciemment et involontairement, à cause de l'absence d'une bonne connaissance des règles (Corder, 1981, p. 10). Les erreurs découlant du transfert négatif se posent par les contradictions du système de deux langues. Les erreurs en question sont précisées avec la méthode d'analyse contrastive. Le processus d'analyse contrastive consiste à identifier et sélectionner les éléments de deux langues pour une comparaison détaillée ; à comparer les domaines semblables et différents, à supposer les domaines possibles qui donnent lieu aux erreurs (Trawiński, 2005, p. 11). Lado (1957) qui développe l'approche contrastive suggère que le processus d'apprentissage se réalise par la comparaison de la langue étrangère avec la langue maternelle. Lado (1957) qui met en avant que la langue maternelle a des répercussions sur l'acquisition de la langue, relève dans sa théorie que les différences entre deux langues rendent l'acquisition plus difficile alors que les similitudes la facilitent. En outre, les apprenants commettent des erreurs ou des interférences quand ils se trouvent en présence de deux langues qui se différencient. Wagner (1982, p. 219) met aussi en avant que les propriétés de la langue cible qui se diffèrent de la langue maternelle peuvent être apprises plus ou moins difficilement en fonction de propriétés similaires ou identiques, et il précise que la difficulté de l'acquisition devient plus sérieuse lorsque la différence entre deux langues est plus importante. Cependant, d'après la théorie de Weinreich, la xénité des langues peut exciter l'intérêt et la curiosité dans l'apprentissage d'une langue étrangère. Par conséquent, une analyse contrastive sur les propriétés de la langue maternelle et de la langue étrangère permet de déterminer à l'avance les causes des difficultés et des interférences éventuelles. Le processus de l'analyse contrastive se compose des étapes comme la définition de deux langues, la comparaison des domaines similaires et différents, la présupposition des domaines éventuels causés des erreurs et la sélection des éléments de deux langues pour effectuer une comparaison détaillée (Trawiński, 2005, p. 11). Afin de rendre efficace l'apprentissage de la langue étrangère, il faut appliquer les étapes du processus de l'analyse contrastive et déterminer les similitudes et les différences de deux langues. La présente étude a donc pour but d'effectuer une analyse contrastive entre le subjonctif français et turc et de découvrir les causes des difficultés rencontrées par les apprenants universitaire Turcs au cours de l'apprentissage du subjonctif français. 
En effet, un certain nombre de questions sont posées depuis bien longtemps concernant ce sujet : L'apprentissage d'une langue étrangère devient-il plus facile quand l'étudiant trouve l'équivalent des structures de sa langue maternelle dans la langue étrangère apprise ? Les transferts effectués de la langue maternelle ont-ils des répercussions positives ou négatives sur l'apprentissage ? Partant de ces questions générales, nous souhaitons soulever dans cette étude les questions plus particulières concernant la difficulté de l'acquisition du mode subjonctif français par les apprenants Turcs. Pour le savoir, tout d'abord, il faut effectuer une étude contrastive du subjonctif français et turc.

\section{Analyse contrastive du subjonctif}

Un examen contrastif du subjonctif dans les deux langues montre clairement qu'il est différent du point de vue morphologique et fonctionnel. L'étude intitulé « le subjonctif français et le volitif turc » de Bosnalı et Topaloğlu (2015) nous a montré effectivement que, sur le plan morphologique, il n'existe en français que deux morphèmes qui signifient le mode subjonctif du verbe: morphème $\{-\mathrm{i}-\}$ pour les $4^{\mathrm{ème}}$ et $5^{\text {ème }}$ personnes et morphème zéro $\{-\emptyset-\}$ pour les autres personnes. Par contre, en ce qui concerne turc, nous sommes en présence de vingt-deux formes différentes du verbe. Quant à son emploi, nous prélevons dix-huit domaines en français pour une seule forme, alors qu'en turc, il est nettement plus limité, malgré ses différents modes et formes plus nombreux que celui du français. 
Topaloğlu, Y. (2017). Acquisition du Subjonctif par Les Étudiants Turcs. Humanitas, 5(10), 333-361

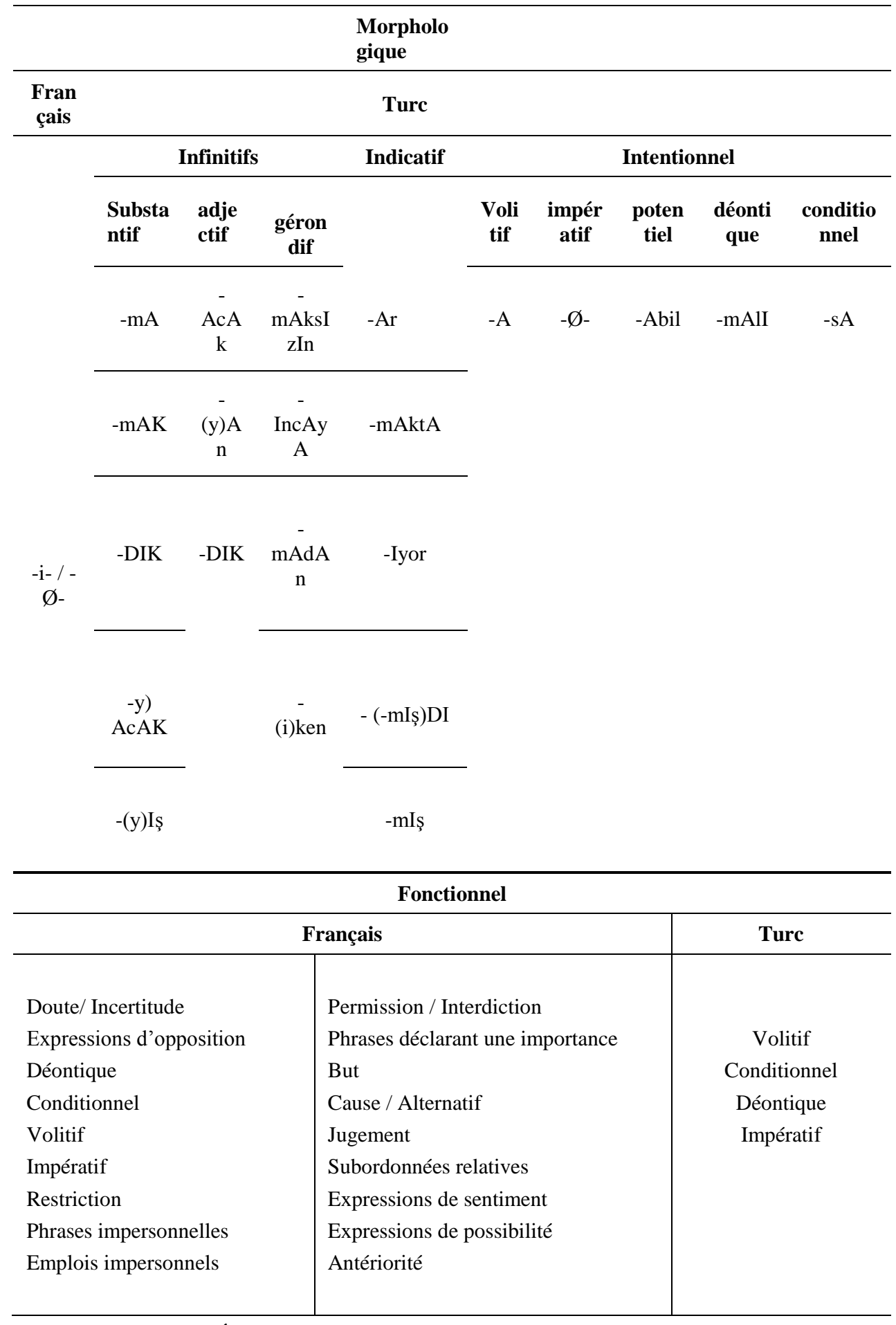

Figure 1. Équivalent morphologique et fonctionnel du subjonctif 
Le subjonctif français est employé dans les phrases impersonnelles et celles-ci ne possèdent pas une phrase de base et sont employées très rarement. Ces phrases peuvent déclarer sémantiquement une supplication, un ordre, une restriction, une exclamation et un désir. On peut employer le subjonctif sans la conjonction «que» mais cet emploi est aussi très rare. Par contre, avec la conjonction «que » et la troisième personne singulier et pluriel, ces phrases possèdent une valeur de l'impératif (Delatour, Jennepin, Léon-Dufour et Teyssier, 2004 ; Cellard, 1996, p. 16), alors qu'en turc, on emploie ces phrases avec des structures différentes.

Le subjonctif français est un mode qui est aussi employé dans les phrases déclarant une importance. Nous rencontrons cette fonction dans les emplois impersonnels ou avec le verbe «importer». Quant au turc, ces structures s'emploient dans les fonctions différentes.

Le subjonctif français possède des domaines d'emploi dans les subordonnées relatives et ce mode dans cette subordonnée se lie à la phrase de base avec les relatifs simples ou composés (Poisson-Quinton, Mimran et Mahéo-Le Coadic, 2002 , p. 245). Par ailleurs, quand la subordonnée précise un but ou une conséquence, on utilise aussi le subjonctif (Dubois et Lagane, 2005, p. 135 ; Cellard, 1996, s. 67). Pourtant, on emploie généralement l'indicatif avec les pronoms relatifs mais quand le pronom utilisé à la place du nom est limité avec le superlatif, on doit employer le subjonctif (Youssef, 2014 ; Cellard, 1996). Par ailleurs, il faut employer le subjonctif quand on utilise l'indéfini ou un pronom indéfini à la place du nom dans ces phrases (Youssef, 2014 ; Cellard, 1996). Quant à cet emploi en turc, on voit que des structures différentes sont équivalentes.

Il existe aussi une fonction du subjonctif dans les phrases qui déclarent une cause ou un alternatif (Dubois et Lagane, 2005) mais en turc, ces structures s'emploient dans les fonctions différentes.

Les expressions de sentiment possèdent un grand domaine d'emploi en français. On emploie le subjonctif plus souvent dans les expressions déclarant un bonheur, un malheur, une satisfaction ou insatisfaction, une émotion, une crainte, un regret, une irritation, un étonnement, un épanouissement, une honte, un désappointement, un souci, une anxiété. Par contre, s'agissant du turc, on peut rencontrer plusieurs domaines d'emplois différents l'un de l'autre.

Le subjonctif français est employé avec les verbes ou les structures déclarant un doute et une certitude, alors qu'en turc, on peut utiliser un mode quelconque équivalent à ces structures.

Un autre domaine d'emploi du subjonctif français est le jugement. Quant au turc, le jugement possède des structures différentes.

Le subjonctif français est employé à la suite des conjonctions qui déclarent le but comme «afin que » ou «pour que» et on peut aussi exprimer le but seulement avec la conjonction «que » (Dubois et Lagane, 2005, p. 142), alors qu'en turc, la déclaration du but est équivalente aux structures différentes. 
Le subjonctif français possède une fonction dans les expressions de possibilité. Quand on exprime une opinion de vraisemblance ou d'invraisemblance, de risque, de chance etc., l'emploi du subjonctif est en question (Cellard, 1996, p. 41-42). Quant au turc, les expressions de possibilité s'emploient avec les structures de l'infinitif ou du verbe potentiel.

Dans les expressions qui restreignent la réalisation d'un fait, on emploie le subjonctif en français (Cellard, 1996), s'agissant du turc, on voit que les gérondifs sont généralement équivalents à cette fonction.

Le subjonctif français est employé à la suite des conjonctions qui possèdent un sens d'opposition, alors qu'en turc, on utilise les infinitifs comme équivalent.

Le subjonctif français est aussi utilisé dans les subordonnées déclarant une interdiction ou une permission, s'agissant du turc, on ne possède pas de telle fonction et les formes différentes peuvent être équivalentes.

Le subjonctif français est aussi employé à la suite des conjonctions qui déclarent l'antériorité (Dubois et Lagane, 2005, s. 140), alors qu'en turc, on utilise généralement les gérondifs comme équivalent.

On peut voir les emplois impersonnels dans toutes les fonctions du subjonctif français mais parfois sémantiquement les autres emplois ne comprennent pas cette fonction. Donc, il faut considérer cette fonction comme un domaine d'emploi séparé. En turc, on peut utiliser une forme quelconque comme équivalent à cette fonction du subjonctif.

Le déontique est indiqué avec le subjonctif dans les subordonnées en français (Cellard, 1996, p. 22) et c'est l'un de l'emploi que l'on rencontre le plus souvent en français. Par ailleurs, dans les phrases qui sont formées avec les structures ou les verbes déclarant le déontique, on emploie plus souvent le subjonctif. Quant au turc, le déontique est équivalent à la fonction déontique du subjonctif français mais le déontique turc est aussi employé pour plusieurs fonctions différentes. Donc, on constate que la fonction déontique du subjonctif français et le déontique turc ne correspondent pas littéralement.

Le subjonctif français est aussi employé dans les phrases qui déclarent une condition et en turc, le conditionnel est équivalent à cette fonction du subjonctif. Le conditionnel turc est aussi employé pour plusieurs fonctions différentes hors de cet emploi. C'est la raison pour laquelle on peut conclure que la fonction conditionnelle du subjonctif français et le conditionnel turc ne correspondent pas littéralement.

Le subjonctif français est aussi employé dans toutes les subordonnées de jugement individuel qui déclarent un désir, un souhait ou une volonté. Il existe aussi une fonction impérative du subjonctif en français qui ne requiert pas une phrase de base et on constitue cette fonction du subjonctif français en employant directement la conjonction «que ». Par contre, s'agissant du turc, il existe le volitif turc et l'impératif comme équivalent à ces deux fonctions du subjonctif mais l'emploi du volitif turc et de l'impératif engendrent la polémique entre les 
linguistes Turcs. Ceux-ci discutent que le volitif turc et l'impératif peuvent être employés l'un à la place de l'autre. On n'arrive pas encore à une explication claire concernant ces deux modes en turc. D'ailleurs, ces deux modes possèdent plusieurs emplois différents en turc. Donc, on peut avancer que la fonction volitive et impérative du subjonctif français et le volitif et l'impératif turc ne correspondent pas littéralement.

Les domaines fonctionnels du subjonctif français sont assez larges et comprennent l'indicatif et l'intentionnel turc et tous les infinitifs dans le tableau 1. En conséquence, cette analyse contrastive nous permet de conclure que le subjonctif français et turc sont différents comme morphologique et fonctionnel l'un de l'autre.

\subsection{Problématique}

La présente étude tente de mettre en évidence les difficultés que les apprenants turcs rencontrent au cours de l'apprentissage du mode subjonctif français et d'expliquer ses causes. En effet, il est communément admis que l'acquisition de ce mode constitue l'un des principaux problèmes rencontrés par les apprenants turcs. Nous voulons soulever dans cette étude les questions concernant la difficulté de l'acquisition du subjonctif français par les étudiants universitaires turcs. Qu'est-ce qui se cache effectivement derrière cette difficulté ?

\subsection{Hypothèse}

Nous pouvons donc avancer que le principal facteur sous-jacent de la difficulté rencontrée par les apprenants Turcs au cours de l'apprentissage du subjonctif français résulte de cette différence observée entre ces deux langues. Ce constat nous amène à réactualiser la théorie selon laquelle la xénité de la langue étrangère est un facteur important dans son acquisition. La différence morphologique et fonctionnelle du subjonctif français peut conduire les apprenants universitaires turcs à ne pas comprendre la logique de l'emploi de ce mode. À partir de cette hypothèse, les résultats de la recherche nous permettront de clarifier ce sujet.

\section{Méthode}

Notre étude exige d'analyser des données statistiques, c'est la raison pour laquelle cette étude s'inscrit dans le cadre de l'approche méthodologique quantitative. Dans notre étude, nous avons profité de la technique d'enquête pour obtenir les données. Il est possible de classifier les questions de l'enquête comme factuel, comportement, attitude, opinion-croyance et informationnel (Balc1, 2015, p. 148-149). Dans notre étude, nous avons préféré utiliser les questions factuelles, d'attitudes et d'informationnels en dépendant de notre hypothèse. Par ailleurs, il existe plusieurs types de questions d'enquête comme questions ouvertes, fermées, semi-fermées. Dans cette étude, tenant compte des données que nous visons à obtenir, nous avons préparé des questions ouvertes, fermées et semi-fermées. 
Afin de recueillir des données sur le sujet traité, nous avons mené une enquête auprès de 100 étudiants inscrits en quatrième année dans le Département de langue et littérature françaises des universités Turques. Avant d'appliquer l'enquête, nous avons déterminé le nombre total d'étudiants qui sont inscrits en quatrième année. Au total, il a été constaté qu'il existe 214 étudiants dans ces universités. Quant au nombre total de participants à cette enquête, 100 étudiants de ces universités se sont présentés. Nous avons planifié de comprendre tous les étudiants qui sont au total 214 à cette enquête mais nous avons rencontré des difficultés pour convaincre tous ces étudiants à participer à cette enquête au cours de l'application.

Nous avons soumis à ces étudiants un questionnaire, composé de 37 questions, dans lequel se trouvent 3 questions factuelles, 18 questions sur l'attitude des apprenants à propos du mode subjonctif et 16 questions informationnelles au sujet de l'emploi du mode subjonctif et indicatif. Les 3 premières questions sur l'attitude, visent à déterminer les sujets et les domaines les plus difficiles dans l'apprentissage du français. Les 7 questions suivantes concernent les avis des apprenants sur la relation entre la similitude et la différence linguistique au cours de l'apprentissage du français. Les 6 questions qui les suivent visent à déterminer les attitudes des étudiants vis-à-vis des différences linguistiques. Nous avons consacré 2 questions sur les attitudes des apprenants face à l'emploi du mode subjonctif. Enfin, 10 questions informationnelles sont réservées à l'emploi réel du mode subjonctif des étudiants, qui sont complétés de 6 questions concernant l'emploi réel de l'indicatif.

Afin de déterminer la fréquence des participants et le taux, le taux valide et le taux total de chaque choix, on a effectué une analyse de fréquence moyennant le programme de SPSS «paquet social pour le chercheur en sciences sociales».

\section{Analyse des données}

Les questions de l'enquête concernant les avis et les attitudes des apprenants ont été élaborées du général au spécifique. Avant tout, on se réfère aux attitudes des étudiants pour confirmer si les similitudes ou les différences linguistiques influencent positivement ou négativement l'acquisition de la langue. Ensuite, on a posé des questions concernant la conjugaison, l'utilisation, la similitude ou la différence entre deux langues. On a classifié les questions afin d'assurer la cohérence et de les rendre plus compréhensible. Selon cette classification, les données ainsi obtenues peuvent être analysées sous six titres particuliers: « sujets et domaines perçus comme les plus difficiles », «attitudes des étudiants vis-à-vis des similitudes linguistiques », «attitudes des étudiants concernant les différences linguistiques », "attitudes des étudiants à l'usage du mode subjonctif », «emploi du mode subjonctif » et «emploi de l'indicatif ».

\subsection{Sujets et domaines les plus difficiles}

On a posé 3 questions pour déterminer les sujets et les domaines perçus comme les plus difficiles en français pour les apprenants. Pour ce faire, premièrement, il a été posé une question aux étudiants : "Quel est le plus difficile sujet pour 
vous au cours de l'apprentissage du français ? ». D'après les données obtenues, $1 \%$ des étudiants ont choisi « le futur simple », $0 \%$ « le passé composé », une grande majorité comme $71 \%$ «le subjonctif », $13 \%$ « le conditionnel », $3 \%$ « l'imparfait » et $12 \%$ des apprenants ont précisé la réponse « autre ». Selon ces déclarations, une majorité importante des étudiants comme $71 \%$ ont précisé « le subjonctif » comme le sujet le plus difficile. Les données obtenues nous montrent que le subjonctif est l'un des sujets le plus difficile pour les apprenants au cours de l'apprentissage du français.

Par ailleurs, pour identifier la difficulté de conjugaison et d'emploi de ce mode par les étudiants, il a été posé une question supplémentaire : "Dans quel domaine vous éprouvez la difficulté ? ». Selon les données obtenues, $13 \%$ des étudiants ont précisé « conjugaison », $40 \%$ « emploi », $38 \%$ « conjugaison et emploi » et $9 \%$ « autre ». Le pourcentage de « conjugaison et emploi »a été nivelé aux taux de choix « conjugaison » et « emploi ». Ainsi, il a été déterminé que $32 \%$ des apprenants ont de la difficulté à conjuguer et $59 \%$ à employer ce mode. Quant à $9 \%$ des étudiants, ils ont préféré la réponse " autre ». Ces données obtenues nous montrent que les apprenants n'ont pas de compétence à employer ce mode et la conjugaison de ce mode est difficile pour les étudiants.

En tenant compte de ces deux dernières questions ci-dessus et les déclarations des apprenants que le subjonctif est difficile à apprendre, il a été posé une autre question : "Est-il difficile d'acquérir le subjonctif ? ». D'après les données obtenues, $12 \%$ des apprenants ont précisé que le subjonctif est « très difficile », $67 \%$ « difficile », $1 \%$ « ni en désaccord ni d'accord », $19 \%$ «pas difficile » et $1 \%$ «pas jamais difficile » à acquérir. Une grande majorité des apprenants comme $79 \%$ ont déclaré que le subjonctif est difficile à apprendre et quant à 21 $\%$ des étudiants, ils ont déclaré qu'il n'est pas difficile d'acquérir ce mode.

Tableau 1 : Sujets et domaines perçus comme les plus difficiles

\begin{tabular}{|c|c|c|c|c|c|c|c|}
\hline \multirow[t]{2}{*}{ Q1 } & & $\begin{array}{l}\text { Futur } \\
\text { Simple }\end{array}$ & $\begin{array}{l}\text { Passé } \\
\text { Composé }\end{array}$ & Subjonctif & Conditionnel & Imparfait & Autre \\
\hline & $\%$ & 1 & 0 & 71 & 13 & 3 & 12 \\
\hline \multirow[t]{2}{*}{ Q2 } & & \multicolumn{2}{|c|}{ Conjugaison } & Emploi & $\begin{array}{l}\text { Conjugaison } \\
\text { et Emploi }\end{array}$ & Autre & \\
\hline & $\%$ & & 13 & 40 & 38 & 9 & \\
\hline \multirow[t]{2}{*}{ Q3 } & & \multicolumn{2}{|c|}{ Très difficile } & Difficile & $\begin{array}{c}\text { Ni en } \\
\text { désaccord ni } \\
\text { d'accord }\end{array}$ & $\begin{array}{c}\text { Pas } \\
\text { difficile }\end{array}$ & $\begin{array}{l}\text { Pas } \\
\text { jamais } \\
\text { difficile }\end{array}$ \\
\hline & $\%$ & \multicolumn{2}{|r|}{12} & 67 & 1 & 19 & 1 \\
\hline
\end{tabular}

Selon les données obtenues à partir de ces questions, le mode subjonctif apparait comme le sujet le plus difficile à apprendre. Il a été identifié surtout que les étudiants ont de la difficulté à conjuguer et à employer ce mode. Ces 
deux aspects différents de la compétence linguistique sont à l'origine de la difficulté rencontrée par les étudiants au cours de leur acquisition du mode en question.

\subsection{Similitudes linguistiques}

Suivant la théorie de Lado, selon laquelle la similitude linguistique peut rendre l'acquisition plus difficile et la différence linguistique peut rendre celle-ci plus facile, nous avons voulu mettre en évidence les attitudes des étudiants à ce sujet. Dans le cadre de cette théorie et en tenant compte la xénité du mode subjonctif, nous avons préparé 7 questions.

Tout d'abord, afin de déterminer si la similitude facilite l'acquisition de la langue ou non, nous avons posé une question générale: "La similitude entre deux langues est-elle un facteur facilitant l'acquisition de la langue ? ». D'après les données obtenues, $2 \%$ des étudiants ont déclaré qu'ils ne sont « pas du tout d'accord », $49 \%$ des apprenants sont « d'accord » et $49 \%$ « tout à fait d'accord ». Une majorité importante des étudiants comme $98 \%$ ont déclaré que la similitude linguistique entre deux langues facilite l'acquisition de la langue.

À partir de cette question ci-dessus, nous avons voulu mettre en évidence les attitudes des étudiants à savoir, en posant une question, s'ils considèrent que le subjonctif français est similaire ou différent du point morphologique et fonctionnel à un mode quelconque dans leur langue maternelle. Selon les données obtenues, $10 \%$ des apprenants ont déclaré que le subjonctif français est « certainement différent », $39 \%$ « diffèrent », $17 \%$ « ni en désaccord ni d'accord », $32 \%$ « similaire » et $2 \%$ « certainement similaire ». D'après ces données obtenues, $49 \%$ des étudiants considèrent que le subjonctif français est différent et $34 \%$ des apprenants pensent que le subjonctif français est similaire morphologique et fonctionnel à un mode quelconque dans leur langue maternelle. Quasi la moitié des étudiants considère que le subjonctif français est différent à un mode quelconque dans leur langue maternelle.

À partir de cette question ci-dessus, nous avons posé une question supplémentaire : " $\mathrm{Si}$ vous dites « similaire » ou « certainement similaire », précisez auquel mode ? » à ceux qui précisent que le subjonctif français est « similaire » ou « certainement similaire ». Nous leur avons demandé d'écrire le mode qui est similaire. Ainsi, nous avons voulu mettre en évidence la cohérence entre ces deux questions et déterminer quel mode considèrent-t-ils étant similaire dans leur langue maternelle. Il n'y a que 26 apprenants, de taux de 34 $\%$ qui ont déclaré qu'il est similaire dans la question précédente, ont répondu cette question. Ces étudiants ont précisé plusieurs modes différents qui existent dans leur langue maternelle. Par contre, on constate qu'avec une grande majorité, $74 \%$ des apprenants ne savent pas si le subjonctif a une équivalence morphologique et fonctionnelle dans leur langue maternelle.

Les apprenants peuvent considérer le subjonctif français comme similaire à une structure ou différent d'une structure existant dans leur langue maternelle. Toutefois, une majorité importante des apprenants représentant un taux de $98 \%$ 
démontrent que la similitude linguistique entre deux langues est un facteur très important dans l'acquisition de la langue. C'est la raison pour laquelle nous avons voulu déterminer "si le subjonctif français est similaire à un mode quelconque dans leur langue maternelle, cette similitude facilite-elle l'apprentissage ou non ? ». Selon les données obtenues, $2 \%$ des apprenants ont déclaré que la similitude rend l'apprentissage « certainement plus difficile», 8 $\%$ «plus difficile », $4 \%$ « ni en désaccord ni d'accord », $64 \%$ «plus facile » et $22 \%$ « certainement plus facile ». Avec $86 \%$ une majorité importante des étudiants considèrent que la similitude est un facteur très important dans l'apprentissage de ce mode tandis que $10 \%$ des apprenants pensent que la différence rend l'apprentissage difficile et $4 \%$ n'ont aucune idée.

$86 \%$ des étudiants déclarent que la similitude morphologique et fonctionnelle de ce mode facilite son apprentissage. Par contre, ces étudiants ont déjà déterminé que le subjonctif est le sujet le plus difficile à acquérir. D'ailleurs, ils ont postulé que la conjugaison du verbe et l'emploi de ce mode sont très difficiles pour eux. C'est la raison pour laquelle nous avons voulu vérifier si ces obstacles de l'apprentissage de ce mode est la conséquence de l'absence de l'équivalence morphologique et fonctionnelle du subjonctif dans leur langue maternelle. Ainsi, il a été présenté la question: «Le subjonctif a-t-il un équivalent dans votre langue maternelle ? ». Selon les données obtenues, $13 \%$ des apprenants ont déclaré qu'il n'a « certainement pas équivalent », $24 \%$ «pas équivalent », $16 \%$ des étudiants sont «ni en désaccord ni d'accord », $24 \%$ des apprenants pensent qu'il a « équivalent » et $23 \%$ « certainement équivalent ». Quand on analyse les différences entre les données obtenues de cette question, on peut conclure que les apprenants ne sont pas sûrs si le subjonctif français a un équivalent structurel dans leur langue maternelle.

À partir de cette question ci-dessus, on a développé une question supplémentaire pour ceux qui ont déclaré, dans la question précédente, que le subjonctif français est «équivalent» et « certainement équivalent». Ainsi, il a été posé la question: "Si le subjonctif français a un équivalent dans votre langue maternelle, ce sont lesquels ? ». 36 apprenants ont répondu à cette question et ils ont précisé plusieurs modes différents.

L'analyse contrastive que nous avons déjà effectuée entre le mode subjonctif français et turc nous montre que le mode subjonctif français n'a pas littéralement d'équivalent en turc. Toutefois, quelques emplois de l'intentionnel turc qui sont le volitif, le conditionnel, le déontique et l'impératif ont la similitude de quelques aspects. Par contre, l'intentionnel turc a plusieurs emplois différents dans son domaine d'utilisation. C'est la raison pour laquelle on a précisé que le mode subjonctif français et l'intentionnel turc sont différents l'un de l'autre. Nous avons préparé une question : «Quel est le mode équivalent du mode subjonctif français en turc ?». Les données obtenues nous montrent que $14 \%$ des apprenants ont affirmé « tout » et $7 \%$ des étudiants ont choisi « aucun ». Le pourcentage de « tout » a été également partagé aux quatre modes. Ainsi, par 77,5\% une majorité importante ont successivement déclaré « le 
volitif », 25,5\% le déontique, $16,5 \%$ le conditionnel et 7,5\% l'impératif. $5 \%$ étudiants ont préféré la réponse «autre ». On voit que le volitif est le plus souvent indiqué pour l'équivalent du subjonctif.

On a déjà analysé que les livres de grammaire préparés en turc associent le mode subjonctif français au volitif turc. Pour déterminer que les apprenants mettent en corrélation effectivement les domaines d'utilisation de deux modes, nous avons préparé cette question : " Les domaines d'emploi du mode subjonctif correspondent-ils à ceux du volitif turc ? ». Les données obtenues nous montrent que $4 \%$ des apprenants indiquent qu'ils ne sont «pas du tout d'accord », $11 \%$ « pas d'accord », $21 \%$ « ni en désaccord ni d'accord » et $59 \%$ des étudiants déclarent qu'ils sont « d'accord» et $5 \%$ 《 tout à fait d'accord». Les données déjà obtenues concernant la question de l'intentionnel turc et ces données ci-dessus sont en cohérence. Il faut ajouter que les livres de grammaire préparés en turc ont une importante influence sur l'association de ces deux modes.

Les apprenants éprouvent aussi de la difficulté pour le transfert du mode subjonctif français dans leur langue maternelle. Il a été posé la question : " Quand vous transférez le mode subjonctif français dans votre langue maternelle, trouvez-vous facilement un équivalent ? ». À partir des données, on déduit que $14 \%$ des apprenants ne sont «pas du tout d'accord », $49 \%$ «pas d'accord », 8 $\%$ « ni en désaccord ni d'accord», $27 \%$ des étudiants sont «d'accord» et $2 \%$ « tout à fait d'accord».

Tableau 2 : Attitudes des étudiants vis-à-vis des similitudes

\begin{tabular}{|c|c|c|c|c|c|c|}
\hline \multirow[t]{2}{*}{ Q4 } & & $\begin{array}{l}\text { Pas du tout } \\
\text { d'accord }\end{array}$ & $\begin{array}{c}\text { Pas } \\
\text { d'accord }\end{array}$ & $\begin{array}{c}\text { Ni en } \\
\text { désaccord } \\
\text { ni d'accord }\end{array}$ & D'accord & $\begin{array}{l}\text { Tout à fait } \\
\text { d'accord }\end{array}$ \\
\hline & $\%$ & 0 & 2 & 0 & 49 & 49 \\
\hline \multirow[t]{2}{*}{ Q5 } & & $\begin{array}{l}\text { Certainement } \\
\text { diffèrent }\end{array}$ & Diffèrent & $\begin{array}{l}\text { Ni en } \\
\text { désaccord } \\
\text { ni d'accord }\end{array}$ & Similaire & $\begin{array}{l}\text { Certainement } \\
\text { similaire }\end{array}$ \\
\hline & $\%$ & 10 & 39 & 17 & 32 & 2 \\
\hline \multirow[t]{2}{*}{ Q6 } & & $\begin{array}{l}\text { Certainement } \\
\text { plus difficile }\end{array}$ & Plus difficile & $\begin{array}{l}\mathrm{Ni} \text { en } \\
\text { désaccord } \\
\text { ni d'accord }\end{array}$ & Plus facile & $\begin{array}{l}\text { Certainement } \\
\text { plus facile }\end{array}$ \\
\hline & $\%$ & 2 & 8 & 4 & 64 & 22 \\
\hline \multirow[t]{2}{*}{ Q7 } & & $\begin{array}{l}\text { Certainement } \\
\text { pas équivalent }\end{array}$ & $\begin{array}{c}\text { Pas } \\
\text { équivalent }\end{array}$ & $\begin{array}{c}\text { Ni en } \\
\text { désaccord } \\
\text { ni d'accord }\end{array}$ & Équivalent & $\begin{array}{l}\text { Certainement } \\
\text { équivalent }\end{array}$ \\
\hline & $\%$ & 13 & 24 & 16 & 24 & 23 \\
\hline Q8 & & Volitif & Conditionnel & Déontique & Impératif & Tout Aucun \\
\hline
\end{tabular}


Topaloğlu, Y. (2017). Acquisition du Subjonctif par Les Étudiants Turcs. Humanitas, 5(10), 333-361

\begin{tabular}{|c|c|c|c|c|c|c|}
\hline & $\%$ & 74 & 13 & 22 & 4 & 14 \\
\hline \multirow[t]{2}{*}{ Q9 } & & $\begin{array}{c}\text { Pas du tout } \\
\text { d'accord }\end{array}$ & $\begin{array}{c}\text { Pas } \\
\text { d'accord }\end{array}$ & $\begin{array}{c}\text { Ni en } \\
\text { désaccord } \\
\text { ni d'accord }\end{array}$ & $D^{\prime}$ accord & $\begin{array}{c}\text { Tout à fait } \\
\text { d'accord }\end{array}$ \\
\hline & $\%$ & 4 & 11 & 21 & 59 & 5 \\
\hline \multirow[t]{2}{*}{ Q10 } & & $\begin{array}{c}\text { Pas du tout } \\
\text { d'accord }\end{array}$ & $\begin{array}{c}\text { Pas } \\
\text { d'accord }\end{array}$ & $\begin{array}{c}\text { Ni en } \\
\text { désaccord } \\
\text { ni d'accord }\end{array}$ & $D^{\prime}$ accord & $\begin{array}{c}\text { Tout à fait } \\
\text { d'accord }\end{array}$ \\
\hline & $\%$ & 14 & 49 & 8 & 27 & 2 \\
\hline
\end{tabular}

Concernant les similitudes linguistiques, il s'avère que la similitude entre deux langues est un facteur très important dans l'acquisition de la langue, la similarité structurelle facilitant l'acquisition de la langue. Par ailleurs, le mode subjonctif est considéré par les étudiants comme un élément de xénité. En effet, près de la moitié des étudiants estiment que celui-ci est différent dans leur langue maternelle. Il ressort d'autre part qu'une grande majorité des étudiants ne savent pas si le subjonctif a une équivalence morphologique et fonctionnelle dans leur langue maternelle. Il faut ajouter que l'intentionnel turc est perçu par les étudiants comme étant équivalent au subjonctif français. Parmi eux, c'est le volitif qui est le plus souvent indiqué pour l'équivalent du subjonctif.

\subsection{Différences linguistiques}

Suivant la théorie de Weinreich, selon laquelle la différence linguistique peut inciter l'intérêt et la curiosité des apprenants, nous avons visé de connaître clairement les attitudes des étudiants à propos de ce sujet. Premièrement, il a été posé la question: "Si une langue étrangère est différente de votre langue maternelle, incitez-vous à apprendre cette langue cible ? ». Les données obtenues nous montrent que $2 \%$ des apprenants ne sont «pas du tout d'accord », $18 \%$ « pas d'accord », $9 \%$ « ni en désaccord ni d'accord », $62 \%$ des étudiants sont «d'accord » et $9 \%$ 《tout à fait d'accord». Par une grande majorité, $71 \%$ des apprenants considèrent que la différence entre deux langues les incite à apprendre une langue étrangère. Selon ces résultats, on peut avancer que la différence entre les langues éveille la curiosité chez les étudiants à acquérir une langue étrangère.

À partir de cette question ci-dessus, on a pensé à poser une question pour déterminer si le subjonctif français est considéré comme un caractère de xénité. Si les étudiants considèrent que le subjonctif français est un élément de xénité, il faut que ce mode éveille la curiosité chez les apprenants selon la théorie de Weinreich. Afin de déterminer les attitudes des étudiants, on a posé la question : «Le subjonctif français est un élément de xénité pour vous ? ». Les données obtenues nous indiquent que $15 \%$ des apprenants considèrent que ce mode est « certainement xénité », $53 \%$ « xénité », $0 \%$ «ni en désaccord ni d'accord », 28 $\%$ des étudiants pensent que celui-ci n'est «pas xénité » et $4 \%$ « certainement pas xénité ». $68 \%$ des étudiants indiquent que le mode subjonctif français 
constitue un caractère de xénité pour eux. Dans la question précédente, les apprenants déclarent que la différence les incite à apprendre une langue étrangère. Le mode subjonctif français est un caractère de xénité pour eux, C'est la raison pour laquelle il faut que ce mode éveille la curiosité chez eux.

À partir de la question ci-dessus, il a été posé une question pour confirmer si la xénité du mode subjonctif éveille la curiosité chez les étudiants. Ainsi, nous avons posé la question : "Si le subjonctif français est un caractère de xénité pour vous, elle vous éveille à apprendre ce mode ? ». Les données obtenues nous indiquent que $13 \%$ des apprenants déclarent que la xénité de ce mode n'a " pas du tout éveillé », $52 \%$ «pas éveillé », $5 \%$ des étudiants ne sont «ni en désaccord ni d'accord », $29 \%$ des apprenants ont indiqué que cette xénité a « éveillé » et a « tout à fait éveillé » leur curiosité à apprendre ce mode. On peut constater que la xénité du mode subjonctif français influence négativement les étudiants à apprendre ce mode. Par ailleurs, on peut avancer que la différence entre les langues n'incite pas toujours la curiosité à acquérir une langue étrangère.

Quant à la conjugaison du verbe du subjonctif français, la conjugaison du verbe de ce mode est différente de la conjugaison de l'indicatif et du conditionnel. Cette différence est aussi un obstacle à acquérir le subjonctif français. Afin de confirmer effectivement si la conjugaison du mode subjonctif est un obstacle pour les apprenants, il a été posé une question : «Est-il difficile de conjuguer le mode subjonctif en français? ». Les données obtenues nous montrent que $19 \%$ des apprenants déclarent qu'il est « très difficile », $60 \%$ « difficile », $3 \%$ des étudiants ne sont «ni en désaccord ni d'accord », $17 \%$ des apprenants indiquent qu'il est « facile » et $1 \%$ « très facile ». Une majorité importante des apprenants comme $79 \%$ déclarent qu'il est difficile de conjuguer le verbe au subjonctif. Les données obtenues déjà concernant les sujets et les domaines perçus comme les plus difficiles et celles sur la conjugaison du verbe du mode subjonctif français sont assez cohérentes.

À partir de la question ci-dessus, nous avons voulu mettre en évidence les attitudes des apprenants pour déterminer à quel point la difficulté de la conjugaison du subjonctif influence les étudiants pendant l'apprentissage de ce mode. Il a été posé une question : «La difficulté de la conjugaison du subjonctif réduit-elle votre curiosité à apprendre ce mode ? ». Les données obtenues nous indiquent que $4 \%$ des apprenants choisissent «pas du tout réduit», $19 \%$ «pas réduit », $8 \%$ des étudiants ne sont « ni en désaccord ni d'accord », $56 \%$ des apprenants choisissent « réduit » et $13 \%$ « tout à fait réduit ». Une grande majorité des étudiants comme $69 \%$ déclarent que la difficulté de la conjugaison du subjonctif réduit leur curiosité à apprendre ce mode. Ainsi, on peut avancer que la différence et la difficulté de la conjugaison de ce mode influencent négativement les apprenants à acquérir ce mode.

Ces résultats ci-dessus nous conduisent effectivement à déterminer si les apprenants peuvent facilement conjuguer le verbe au subjonctif. C'est la raison 
Topaloğlu, Y. (2017). Acquisition du Subjonctif par Les Étudiants Turcs. Humanitas, 5(10), 333-361

pour laquelle on a préparé la question : «Pouvez-vous facilement conjuguer le verbe au subjonctif ? ». Les données obtenues nous indiquent que $19 \%$ des apprenants déclarent qu'ils ne sont «pas du tout d'accord», $33 \%$ «pas d'accord », $25 \%$ « ni en désaccord ni d'accord », $20 \%$ des étudiants indiquent qu'ils sont « d'accord » et $3 \%$ « tout à fait d'accord ». Selon ces données, plus de la moitié des apprenants ont de la difficulté à conjuguer le verbe de ce mode en question.

Tableau 3 : Attitudes des étudiants concernant les différences

\begin{tabular}{|c|c|c|c|c|c|c|}
\hline \multirow[t]{2}{*}{ Q11 } & & $\begin{array}{c}\text { Pas du tout } \\
\text { d'accord }\end{array}$ & $\begin{array}{c}\text { Pas } \\
\text { d'accord }\end{array}$ & $\begin{array}{c}\text { Ni en } \\
\text { désaccord ni } \\
\text { d'accord }\end{array}$ & $D^{\prime}$ accord & $\begin{array}{l}\text { Tout à fait } \\
\text { d'accord }\end{array}$ \\
\hline & & 2 & 18 & 9 & 62 & 9 \\
\hline \multirow[t]{2}{*}{ Q12 } & $\%$ & $\begin{array}{c}\text { Certainement } \\
\text { xénité }\end{array}$ & Xénité & $\begin{array}{c}\text { Ni en } \\
\text { désaccord ni } \\
\text { d'accord }\end{array}$ & $\begin{array}{l}\text { Pas } \\
\text { xénité }\end{array}$ & $\begin{array}{c}\text { Certainement } \\
\text { pas xénité }\end{array}$ \\
\hline & & 15 & 53 & 0 & 28 & 4 \\
\hline \multirow[t]{2}{*}{ Q13 } & $\%$ & $\begin{array}{l}\text { Pas du tout } \\
\text { éveillé }\end{array}$ & Pas éveillé & $\begin{array}{c}\text { Ni en } \\
\text { désaccord ni } \\
\text { d'accord }\end{array}$ & éveillé & $\begin{array}{l}\text { Tout à fait } \\
\text { éveillé }\end{array}$ \\
\hline & & 13 & 52 & 5 & 29 & 1 \\
\hline \multirow[t]{2}{*}{ Q14 } & & Très difficile & Difficile & $\begin{array}{c}\text { Ni en } \\
\text { désaccord ni } \\
\text { d'accord }\end{array}$ & Facile & Très facile \\
\hline & $\%$ & 19 & 60 & 3 & 17 & 1 \\
\hline \multirow[t]{2}{*}{ Q15 } & & $\begin{array}{l}\text { Pas du tout } \\
\text { réduit }\end{array}$ & Pas réduit & $\begin{array}{c}\text { Ni en } \\
\text { désaccord ni } \\
\text { d'accord }\end{array}$ & réduit & $\begin{array}{l}\text { Tout à fait } \\
\text { réduit }\end{array}$ \\
\hline & $\%$ & 4 & 19 & 8 & 56 & 13 \\
\hline \multirow[t]{2}{*}{ Q16 } & & $\begin{array}{l}\text { Pas du tout } \\
\text { d'accord }\end{array}$ & $\begin{array}{c}\text { Pas } \\
\text { d'accord }\end{array}$ & $\begin{array}{c}\text { Ni en } \\
\text { désaccord ni } \\
\text { d'accord }\end{array}$ & D'accord & $\begin{array}{l}\text { Tout à fait } \\
\text { d'accord }\end{array}$ \\
\hline & $\%$ & 19 & 33 & 25 & 20 & 3 \\
\hline
\end{tabular}

Il a été constaté effectivement que les différences linguistiques jouent un rôle important dans la motivation des étudiants. Toujours est-il que, bien que le subjonctif soit considéré comme une xénité, il ne suscite pas la curiosité chez les étudiants. Cette deuxième donnée spécifique sur le subjonctif apparait contradictoire de la première qui est sur la xénité en général. En effet, la xénité du mode subjonctif est un facteur négatif dans leur apprentissage. On peut donc avancer que la diversité linguistique n'est pas toujours une source d'intérêt et de curiosité. La conjugaison du subjonctif est aussi estimée très difficile par les étudiants. En outre, les étudiants déclarent qu'ils ne trouvent pas l'équivalent 
des domaines d'utilisation du mode subjonctif dans leur langue maternelle ; ce qui entraine une difficulté supplémentaire en ce qui concerne l'acquisition de ce mode.

\subsection{Usage du subjonctif : attitudes}

Il a été posé deux questions concernant les attitudes des étudiants à l'usage du mode subjonctif. Nous prélevons dix-huit domaines d'emploi en français pour le subjonctif. Il est important qu'on sache bien les domaines d'emploi du subjonctif pour pouvoir bien employer ce mode. C'est en partant de ce constat qu'on a posé la question : «Connaissez-vous bien tous les domaines d'emploi du subjonctif?». Les données obtenues nous indiquent que $25 \%$ des apprenants déclarent qu'ils ne sont «pas du tout d'accord », $44 \%$ «pas d'accord »,23\% « ni en désaccord ni d'accord », $8 \%$ des étudiants sont « d'accord » et 0 $\%$ « tout à fait d'accord ». Une grande majorité des apprenants considèrent qu'ils ne connaissent pas bien tous les domaines d'emploi du subjonctif. Les données obtenues jusque-là à propos des sujets et des domaines perçus comme les plus difficiles et celles sur les domaines d'emploi du subjonctif français sont assez cohérents.

Par ailleurs, il faut bien saisir le cadre sémantique du subjonctif afin d'apprendre bien ce mode. Il a été posé la question ainsi : "Comprenez-vous bien la logique d'emploi du subjonctif? » pour qu'on détermine effectivement que les apprenants connaissent bien les domaines d'emploi de ce mode. Les données obtenues nous indiquent que $6 \%$ des étudiants expriment qu'ils ne sont «pas du tout d'accord », $37 \%$ «pas d'accord », $1 \%$ « ni en désaccord ni d'accord », $51 \%$ des apprenants déclarent qu'ils sont « d'accord » et $5 \%$ « tout à fait d'accord ». $56 \%$ des étudiants signalent qu'ils comprennent bien la logique d'emploi du subjonctif alors que $43 \%$ des apprenants expriment qu'ils ne savent pas ces domaines d'emploi. Par contre, dans la question précédente une majorité importante des étudiants déclarent qu'ils ne connaissent bien les domaines d'emploi du subjonctif. Donc, les réponses dans ces deux questions ne sont pas cohérentes.

Nous avons voulu mettre en évidence les idées des étudiants à propos de ce sujet avec la dernière question de cette enquête concernant les attitudes des apprenants. Cette question a été préparée de façon ouverte. Cette question n'a été répondue que par neuf étudiants. Par ailleurs, les données obtenues n'ont pas concerné notre sujet traité. C'est la raison pour laquelle nous n'avons pas pris en considération les données issues de cette question.

Tableau 4 : Attitudes des étudiants à l'usage du mode subjonctif

\begin{tabular}{|c|c|c|c|c|c|c|}
\hline \multirow[t]{2}{*}{ Q17 } & & $\begin{array}{c}\text { Pas du tout } \\
\text { d'accord }\end{array}$ & Pas d'accord & $\begin{array}{c}\text { Ni en } \\
\text { désaccord } \\
n i \\
\text { d'accord }\end{array}$ & D'accord & $\begin{array}{c}\text { Tout à } \\
\text { fait } \\
\text { d'accord }\end{array}$ \\
\hline & $\%$ & 25 & 44 & 23 & 8 & 0 \\
\hline
\end{tabular}


Topaloğlu, Y. (2017). Acquisition du Subjonctif par Les Étudiants Turcs. Humanitas, 5(10), 333-361

\begin{tabular}{cccccc}
\hline Q18 & $\begin{array}{c}\text { Pas du tout } \\
\text { d'accord }\end{array}$ & Pas d'accord & $\begin{array}{c}\text { Ni en } \\
\text { désaccord } \\
n i \\
\text { d'accord }\end{array}$ & D'accord & $\begin{array}{c}\text { Tout à } \\
\text { fait } \\
\text { d'accord }\end{array}$ \\
\hline$\%$ & 6 & 37 & 1 & 51 & 5 \\
\hline
\end{tabular}

Ce mode difficile à transférer dans leur langue maternelle, n'est pas très bien connu quant aux domaines d'emploi. En effet, quoique plus de la moitié d'entre eux déclarent qu'ils comprennent la logique d'utilisation du subjonctif. Il faut donc prendre en considération également des pratiques réelles des étudiants afin de déterminer si ces données reflètent la vérité.

\subsection{Usage du subjonctif : emplois}

Il existe dix-huit domaines d'emploi du subjonctif mais prenant en considération les emplois les plus rencontrés au cours de l'apprentissage, dans les livres de grammaire et d'exercice, nous avons choisi dix emplois les plus utilisés. Ces dix emplois en question sont successivement «le volitif», « l'incertitude », « la possibilité », « le doute », « l'opposition », « la restriction », «le déontique », « le conditionnel », « l'impératif » et « le but».

En premier lieu, nous avons présenté une phrase : «Je souhaite / je voudrais / j'ai envie que vous ....... ma chambre » afin que les apprenants achèvent avec un mode accordant. Il existe plusieurs verbes comme « souhaiter », « vouloir» et « avoir envie » ou emplois qui nécessitent l'utilisation du subjonctif. Nous avons choisi ces verbes parce que les étudiants les emploient plus souvent au cours de l'apprentissage et ils rencontrent ceux-ci plus fréquemment dans les livres de grammaire et d'exercices. Les données obtenues nous indiquent que 47 $\%$ des apprenants ont déclaré la bonne réponse « rangiez » alors que $53 \%$ des étudiants ont choisi « rangez », « rangerez », « rangeriez » et « aviez rangé ». Il a été constaté que plus de la moitié des apprenants n'amènent pas à employer la fonction volitive du subjonctif.

Les subordonnées relatives en français nécessitent l'emploi du subjonctif quand l'incertitude est en question. Les expressions d'incertitude se composent des articles indéfinis ou négatifs comme «un », « une », « des », « rien », « aucun », « pas un ». Pour confirmer si les apprenants ont de la difficulté à choisir le mode dans les subordonnées relatives quand il s'agit de l'incertitude, nous avons présenté la phrase : "Je cherche une fille qui ........... trois langues ». Les données obtenues nous indiquent que $16 \%$ des apprenants ont déclaré le bon choix qui est « connaisse » tandis qu'une majorité importante des étudiants comme $84 \%$ ont choisi les mauvaises réponses : « connaît », « connaîtra », « avait connu ", " connaîtrait ». Ainsi, il est constaté que l'expression d'incertitude dans les subordonnées relatives constitue effectivement une difficulté majeure pour les apprenants.

Les expressions de possibilité entraînent l'emploi du subjonctif et les apprenants rencontrent celles-ci plus souvent au cours de l'apprentissage du 
français. Il existe plusieurs structures de possibilité en français qui exigent le subjonctif. Nous avons préféré la structure «il est possible que » vu que les apprenants emploient cette structure plus souvent au cours de l'apprentissage du français et ils rencontrent celle-ci plus fréquemment dans les livres de grammaire et d'exercices. Afin de préciser si les apprenants peuvent facilement employer cette structure, nous avons présenté une phrase : «Il est possible qu'elle ........ malade ». Les données obtenues nous montrent que $56 \%$ des apprenants ont déclaré le bon choix qui est « soit» alors que $44 \%$ des étudiants ont choisi les mauvaises réponses qui sont « est », «sera », « va être » et «était ». Selon ces données, on peut ressortir que près de la moitié des étudiants n'ont pas réussi à compléter la phrase avec la bonne réponse.

Le subjonctif français est plus souvent employé dans les expressions de doute. Alors qu'on requiert le subjonctif dans les phases négatives et interrogatives de ces expressions, dans les phases affirmatives on emploie l'indicatif. Par exemple ; l'expression affirmative et certitude comme « c'est sûr qu'il ...... son examen » entraîne l'indicatif tandis que l'expression négative exprimant le doute comme « ce n'est pas sûr qu'il ...... son examen » nécessite le subjonctif. C'est la raison pour laquelle les apprenants ont de la difficulté à employer le mode lorsque la phrase exprime le doute. C'est la raison pour laquelle nous avons présenté une phrase : " ce n'est pas sûr qu'il ...... son examen » pour mettre effectivement en évidence cette difficulté. Nous avons demandé aux apprenants de remplir la bonne réponse. Les données obtenus nous indiquent que $26 \%$ des étudiants ont pu accomplir la bonne réponse qui est « ait réussi » tandis que $74 \%$ des apprenants ont échoué en choisissant les mauvaises réponses qui sont « réussis », «a réussi », « avait réussi » et « réussissait». Ces données nous conduisent à avancer qu'une majorité importante des étudiants ont de la difficulté d'employer cette fonction du subjonctif.

Le déontique est l'une des fonctions les plus fréquemment employées du subjonctif. Il existe plusieurs structures en français qui expriment le déontique comme « il faut », « il est nécessaire / indispensable ». Alors que la structure impersonnelle « il faut » impose l'emploi de l'infinitif, les structures impersonnels " il est nécessaire / indispensable » exigent l'utilisation de la préposition « de ». Quand ces structures en question emploient avec la conjonction «que », celles-ci nécessitent l'emploi du subjonctif. Ces différentes utilisations du déontique représentent une difficulté pour les apprenants. Pour confirmer effectivement cette difficulté, nous avons présenté aux étudiants une phrase : «Il faut (est nécessaire / indispensable) que tu ...... tes devoirs » et leur avons demandé d'accomplir cette phrase avec le bon choix. Les données obtenues de ces emplois nous montrent que $55 \%$ des apprenants arrivent à employer la bonne réponse qui est « fasses » tandis que $45 \%$ des étudiants, très près de la moitié, ont préféré les mauvais choix qui sont «fais », «ferais », « faisais », «feras ». Il a été constaté que très près de la moitié des apprenants ont échoué à employer cette structure du subjonctif. 
Le subjonctif est aussi employé dans la fonction de l'impératif. Il existe aussi l'impératif en turc et quelques emplois de l'impératif turc s'est vu comme l'équivalent du subjonctif. Par ailleurs, il existe le mode impératif en français. Nous avons voulu mettre en évidence les pratiques des apprenants à ce sujet en préparant une phrase en turc: "Yaşayasın! 》. Les données obtenues nous indiquent que $60 \%$ des apprenants ont choisi la bonne réponse qui est « que tu vives ! » alors que $40 \%$ des étudiants ont déclaré les mauvais choix qui sont « tu vivras », «tu vis », « tu vivrais » et « vis ! ». Il a été constaté que $40 \%$ des étudiants ont de la difficulté à employer cette fonction du subjonctif.

Il existe plusieurs subordonnées conjonctives en français qui expriment la cause, la conséquence, le but, le temps, l'opposition, la restriction, la condition et la supposition. Les apprenants ont du mal à employer le mode dans les subordonnées conjonctives. Afin de révéler cette difficulté, nous avons préparé une question à propos de ces subordonnées conjonctives en choisissant les conjonctions les plus rencontrées ce qui expriment l'opposition, la restriction, la condition et le but au cours de l'apprentissage du français, dans les livres de grammaire et d'exercices.

D'un côté, nous avons posé une question concernant la conjonction de l'opposition. Nous avons présenté une phrase : "Il est parti bien que / quoiqu'il ...... » et avons demandé aux apprenants de terminer cette phrase avec le bon choix. Les données obtenues nous montrent que $45 \%$ des étudiants sont arrivés à terminer cette phrase avec le bon choix qui est «pleuve» alors que $55 \%$ des apprenants ont choisi les mauvaises réponses qui sont «pleut», «pleuvait», «a plu », «avait plu». Il a été conclu que plus de la moitié des apprenants ne sont pas compétents pour employer cette conjonction de l'opposition du subjonctif.

D'un autre côté, nous avons présenté la conjonction de la restriction « jusqu'à ce que » qui requiert le subjonctif. Comme une préposition « jusqu'à » exige un nom à la suite mais quand on ajoute la conjonction « ce que », celle-ci impose l'emploi du subjonctif. C'est la raison pour laquelle les apprenants peuvent parfois ne pas employer le bon mode à la suite de la préposition « jusqu'à » et de la conjonction « jusqu'à ce que ». Donc, Pour confirmer cette difficulté, nous avons présenté cette phrase : "On ne mangera pas jusqu'à ce que ta mère .... » et avons demandé aux étudiants de compléter cette phrase avec la bonne réponse. Les données obtenues nous indiquent que $44 \%$ des apprenants réagissent vers la bonne réponse qui est « vienne » tandis que $56 \%$ des étudiants ont déclaré les mauvais choix qui sont «viendra », « vient», « est venue », " va venir ». Plus de la moitié des étudiants ont déterminé l'indicatif et cela nous permet de conclure que les apprenants ont de la difficulté pour employer la conjonction de restriction du subjonctif.

Une autre fonction du subjonctif est d'exprimer la condition ; la conjonction « à condition que » exige l'utilisation du subjonctif. Par contre, quand cette conjonction prend par la suite la préposition « de » au lieu de la conjonction « que », celle-ci impose l'emploi de l'indicatif. C'est la raison pour laquelle les 
apprenants peuvent parfois ne pas employer le bon mode à la suite de ces deux fonctions. Pour préciser effectivement cet obstacle, nous avons présenté une phrase : « Il serait bien arrivé à condition qu'il ..... le bon train » et avons demandé aux apprenants d'accomplir avec la bonne réponse. Les données obtenues nous montrent que $34 \%$ des étudiants parviennent à employer la bonne réponse qui est « ait pris » alors que $66 \%$ des apprenants ont choisi les mauvais choix qui sont «a pris », « avait pris », « prenait», « prendrait». Une grande majorité des étudiants comme $66 \%$ ont déclaré l'indicatif et cela nous conduit à conclure que les apprenants ont de la difficulté à employer cette conjonction.

Finalement, nous avons posé une question concernant l'emploi de la conjonction «afin que » qui exprime le but et impose l'utilisation du subjonctif. Comme les autres types de conjonction, quand on ajoute à la suite de cette conjonction la préposition « de » au lieu de la conjonction « que », celle-ci exige l'emploi de l'indicatif. Nous avons donné la phrase : «Elle s'est déguisée afin qu'on ne la .... pas » et nous avons demandé aux apprenants d'achever avec la bonne réponse. Les données obtenues nous indiquent que $42 \%$ des apprenants arrivent à employer le bon choix qui est « reconnaisse », alors que $58 \%$ des étudiants ont déclaré les mauvaises réponses qui sont « reconnaît », « reconnaîtrait », « reconnut » et « reconnaissait ». Plus de la moitié des étudiants ont déterminé l'indicatif et cela nous permet de conclure que les apprenants ne sont pas compétents pour employer cette conjonction.

Tableau 5 : Emploi du mode subjonctif

\begin{tabular}{|c|c|c|c|c|c|c|}
\hline \multirow[t]{2}{*}{ Q19 } & & rangez & rangerez & rangeriez & rangiez & aviez rangé \\
\hline & $\%$ & 17 & 4 & 27 & 47 & 5 \\
\hline \multirow[t]{2}{*}{ Q20 } & & connaît & connaîtra & connaisse & $\begin{array}{l}\text { avait } \\
\text { connu }\end{array}$ & connaîtrait \\
\hline & $\%$ & 77 & 1 & 16 & 4 & 2 \\
\hline \multirow[t]{2}{*}{ Q21 } & & est & sera & soit & va être & était \\
\hline & $\%$ & 27 & 12 & 56 & 1 & 4 \\
\hline \multirow[t]{2}{*}{ Q22 } & & réussis & ait réussi & a réussi & $\begin{array}{l}\text { avait } \\
\text { réussi }\end{array}$ & réussissait \\
\hline & $\%$ & 26 & 26 & 33 & 9 & 6 \\
\hline \multirow[t]{2}{*}{ Q23 } & & fais & ferais & faisais & feras & fasses \\
\hline & $\%$ & 27 & 7 & 5 & 6 & 55 \\
\hline \multirow[t]{2}{*}{ Q24 } & & pleut & pleuvait & a plu & avait plu & pleuve \\
\hline & $\%$ & 28 & 15 & 6 & 6 & 45 \\
\hline Q25 & & viendra & vient & est venue & va venir & vienne \\
\hline
\end{tabular}


Topaloğlu, Y. (2017). Acquisition du Subjonctif par Les Étudiants Turcs. Humanitas, 5(10), 333-361

\begin{tabular}{|c|c|c|c|c|c|c|}
\hline & $\%$ & 20 & 19 & 7 & 10 & 44 \\
\hline \multirow[t]{2}{*}{ Q26 } & & a pris & ait pris & avait pris & prenait & prendrait \\
\hline & $\%$ & 26 & 34 & 16 & 9 & 15 \\
\hline \multirow[t]{2}{*}{ Q27 } & & Tu vivras & Tu vis & Tu vivrais & Vis! & $\begin{array}{c}\text { Que tu vives } \\
!\end{array}$ \\
\hline & $\%$ & 6 & 2 & 11 & 21 & 60 \\
\hline \multirow[t]{2}{*}{ Q28 } & & reconnaît & reconnaîtrait & reconnaisse & reconnut & reconnaissait \\
\hline & & 39 & 5 & 42 & 2 & 12 \\
\hline
\end{tabular}

Selon les données obtenues concernant l'emploi du mode subjonctif, il parait clair que le subjonctif constitue une difficulté majeure pour les apprenants, notamment en raison des divergences de l'emploi du subjonctif dans les deux langues. Les données obtenues à propos des compétences sur l'emploi et celles sur les pratiques sont assez cohérentes.

\subsection{Usage de l'indicatif : emplois}

Nous avons préparé six questions concernant l'emploi de l'indicatif en tenant compte des emplois du subjonctif. Nous avons visé à confirmer le fait que les apprenants sont plus à l'aise dans le domaine de l'indicatif ou du subjonctif.

Tout d'abord, nous avons présenté la structure «il est évident que » qui exprime la certitude et impose l'indicatif et qui s'oppose à l'emploi de la possibilité du subjonctif. Afin de déterminer si les apprenants déclarent l'indicatif ou le subjonctif, nous avons adressé aux étudiants une phrase : «il est évident qu'elle ....... cette faute » et leur avons demandé d'achever cette phrase avec le bon mode. Les données obtenues nous montrent que $50 \%$ des apprenants parviennent à employer le bon choix qui est «a fait »alors que l'autre moitié ont déclaré les mauvaises réponses. On peut conclure que la moitié des étudiants ont réussi à choisir la bonne réponse.

On emploie l'infinitif à la suite de quelques verbes comme « aimer », «pouvoir », « vouloir » et « savoir ». Par contre, ceux-ci exigent l'emploi d'une subordonnée après avoir ajouté la conjonction «que » à la suite de ces verbes. Plusieurs verbes comme « aimer », « pouvoir », « savoir » et « vouloir» imposent l'emploi de l'indicatif ou du subjonctif en dépendant de leurs fonctions ou de leurs valeurs sémantiques. L'un des verbes qui nécessite l'emploi de l'indicatif est le verbe «savoir ». Nous avons préparé une phrase : « Je sais que tu...... » en considérant que les apprenants rencontrent ce verbe plus souvent au cours de l'apprentissage du français. Les données obtenues nous montrent que $70 \%$ des apprenants sont parvenus à déclarer le bon choix qui est « vas bien » tandis que $30 \%$ des étudiants ont choisi les mauvaises réponses qui sont « sois allé(e) bien », «serais allé(e) bien » et «allasses bien ». Il a été constaté qu'une majorité importante des apprenants sont arrivés à choisir le bon mode. 
Comme les subordonnées relatives imposent l'emploi de l'indicatif quand la valeur sémantique de la phrase est définie, nous avons proposé la phrase « je cherche la fille qui ...... trois langues » pour confirmer que si les apprenants distinguent l'emploie du subjonctif et de l'indicatif. Les données obtenues nous montrent que $83 \%$ des apprenants ont déclaré le bon choix qui est « connaît » alors que $17 \%$ des étudiants ont choisi les mauvaises réponses qui sont « ait connu », « connaisse », « eût connu » et « aurait connu ». Il a été conclu que les apprenants sont compétents pour employer cette fonction de l'indicatif.

Nous avons déjà déclaré qu'il existe plusieurs subordonnées conjonctives en français qui expriment la cause, l'antériorité, la conséquence, le but, le temps, l'opposition, la restriction, la condition et la supposition. Les apprenants ont effectivement de la difficulté à employer le bon mode à la suite de ces conjonctions. Afin de confirmer cette difficulté, nous avons préparé des questions concernant la cause, l'antériorité et les conjonctions « étant donné que » et « après que » qui imposent l'emploi de l'indicatif.

La première question était à propos de la conjonction «étant donné que »qui exige l'emploi de l'indicatif et que les apprenants rencontrent plus souvent. Nous avons présenté une phrase : «Tu devrais bien connaitre Paris étant donné que tu $\mathrm{y}$...... déjà allé(e) » et avons demandé aux apprenants d'accomplir cette phrase avec le bon mode. Les données obtenues nous indiquent que $31 \%$ des étudiants sont parvenus à réagir vers le bon choix qui est «es » alors que $69 \%$ des apprenants ont choisi les mauvaises réponses qui sont « eusses », « sois », « serais » et «seras ». $25 \%$ sur $69 \%$ des étudiants qui ont déclaré le mauvais mode sont arrivés à employer l'une des réponses de l'indicatif bien que leurs choix soient faux.

« Avant que » est une conjonction qui exprime l'antériorité et impose l'emploi du subjonctif mais " après que » qui s'oppose à la conjonction " avant que » exige l'emploie de l'indicatif. L'emploi de ces deux différentes conjonctions posent effectivement une difficulté pour les apprenants au cours de l'apprentissage du français. Pour confirmer cette difficulté, nous avons préparé une question: "On regardera un film après que ton ami ...... " et avons demandé aux apprenants de terminer cette phrase avec le bon mode. Les données obtenues nous montrent que $33 \%$ des apprenants ont complété cette phrase avec le bon choix qui est «sera venu » tandis que $67 \%$ des étudiants ont déclaré les mauvaises réponses : «vienne», «vient», « est venu », «étais venu ». Quoiqu'une majorité importante des apprenants comme $77 \%$ sachent qu'ils doivent employer l'indicatif, seulement $33 \%$ des étudiants sont arrivés à réagir vers le bon choix. Cela nous permet de conclure que les apprenants ont aussi la difficulté à employer l'indicatif.

Les fonctions qui expriment le doute imposent l'emploi du subjonctif. Quant à l'indicatif, il existe les fonctions qui révèlent la certitude. La structure « il est certain que » est l'une des fonctions qui expriment la certitude et exige l'emploi de l'indicatif. Les apprenants n'arrivent pas parfois à employer le bon mode à la 
Topaloğlu, Y. (2017). Acquisition du Subjonctif par Les Étudiants Turcs. Humanitas, 5(10), 333-361

suite de ces fonctions. Pour confirmer cet obstacle, nous avons présenté aux étudiants une phrase : "Il est certain que ma mère ..... la cuisine maintenant " et leur avons demandé d'accomplir cette phrase avec le bon mode. Les données obtenues nous indiquent que $53 \%$ des apprenants sont parvenus à compléter cette phrase avec la bonne réponse qui est « fait » alors que $47 \%$ des étudiants ont déclaré les mauvais choix qui sont «fasse », « aurait fait », « ait fait » et « eût fait ». Il a été conclu que plus de la moitié des apprenants savent employer le bon mode dans ces fonctions de la certitude.

Tableau 6 : Emploi de l'indicatif

\begin{tabular}{|c|c|c|c|c|c|c|}
\hline \multirow[t]{2}{*}{ Q29 } & & ait fait & a fait & aurait fait & fasse & fit \\
\hline & $\%$ & 10 & 50 & 5 & 31 & 4 \\
\hline \multirow[t]{2}{*}{ Q30 } & & ailles bien & $\begin{array}{l}\text { sois allé(e) } \\
\text { bien }\end{array}$ & $\begin{array}{c}\text { serais allé(e) } \\
\text { bien }\end{array}$ & vas bien & allasses bien \\
\hline & $\%$ & 16 & 10 & 4 & 70 & 0 \\
\hline \multirow[t]{2}{*}{ Q31 } & & ait connu & connaît & connaisse & $\begin{array}{c}\text { eût } \\
\text { connu }\end{array}$ & aurait connu \\
\hline & $\%$ & 2 & 83 & 12 & 1 & 2 \\
\hline \multirow[t]{2}{*}{ Q32 } & & eusses & es & sois & serais & seras \\
\hline & $\%$ & 2 & 31 & 42 & 16 & 9 \\
\hline \multirow[t]{2}{*}{ Q33 } & & sera venu & vienne & vient & est venu & étais venu \\
\hline & $\%$ & 33 & 23 & 17 & 20 & 7 \\
\hline \multirow[t]{2}{*}{ Q34 } & & fasse & aurait fait & fait & ait fait & eût fait \\
\hline & $\%$ & 32 & 7 & 53 & 6 & 2 \\
\hline
\end{tabular}

Avec les six questions concernant l'indicatif, nous avons souhaité déterminer si les étudiants savaient le distinguer du subjonctif et comparer les compétences des étudiants dans ces deux modes distincts. Les données obtenues confirment que la différence entre ces deux modes n'est pas bien intériorisée, en raison de la complexité des domaines de l'emploi du subjonctif. Nous pouvons constater que les apprenants sont beaucoup plus à l'aise dans le domaine de l'indicatif; et cela est valable aussi bien pour la conjugaison que pour l'emploi. 
Topaloğlu, Y. (2017). Acquisition du Subjonctif par Les Étudiants Turcs. Humanitas, 5(10), 333-361

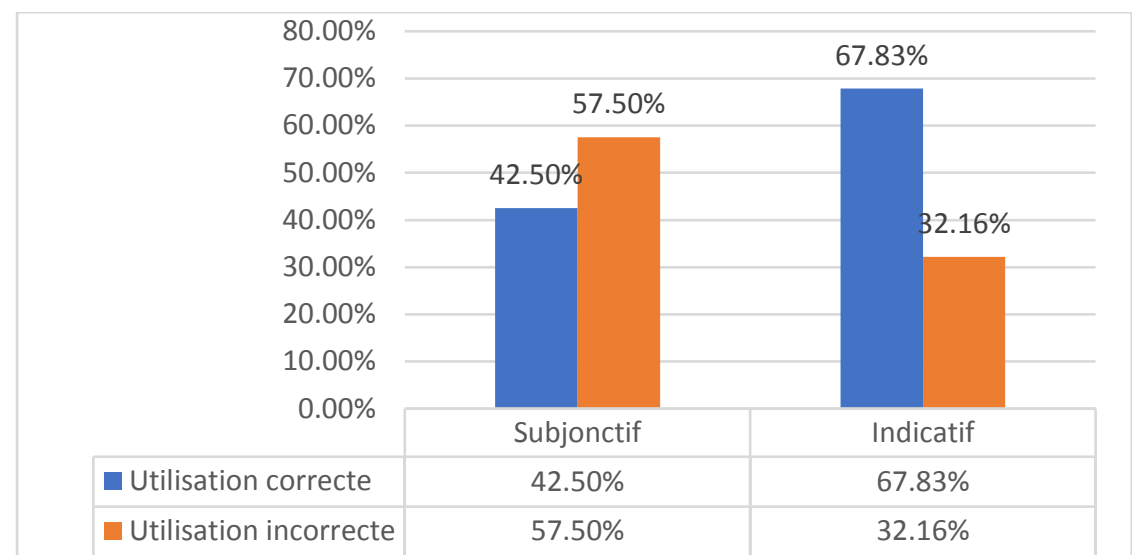

Figure 2. Taux de réussite du mode subjonctif et de l'indicatif

En conséquence, la moyenne arithmétique de l'emploi de deux modes nous indique que le taux de réussite du subjonctif est de $42,50 \%$ alors que le taux de réussite de l'indicatif est de $67,83 \%$. Nous pouvons constater que ce taux de réussite de l'indicatif dépend de la similitude de l'indicatif en français et en turc. Par contre la xénité du subjonctif influence négativement son taux de réussite de l'emploi.

\section{Conclusion}

Les théories de l'acquisition de la langue et les données obtenues à partir de l'analyse contrastive du mode subjonctif en français et en turc nous permettent de mettre en avant l'hypothèse selon laquelle la xénité de ce mode serait à l'origine de la difficulté rencontrée par les étudiants universitaires turcs au cours de leur apprentissage.

Selon les données obtenues concernant les attitudes des apprenants, le subjonctif est l'un des sujets les plus difficiles. On constate que c'est la conjugaison et l'utilisation de ce mode qui sont à l'origine de la difficulté. Il a été déterminé que les étudiants ne sont pas compétents dans l'utilisation du mode subjonctif et que cela constitue un obstacle important. Il a été conclu que le subjonctif, ne possédant pas complètement un caractère de xénité, ne constitue pas non plus un élément commun pour les étudiants turcs. Une majorité importante des étudiants ont effectivement déclaré que le subjonctif français est différent d'une structure dans leur langue maternelle. D'ailleurs, les apprenants ont exprimé qu'ils ne savent pas l'équivalent morphologique et fonctionnel du subjonctif dans leur langue maternelle. Par contre, les étudiants ont précisé plusieurs modes avec l'intentionnel turc comme l'équivalent de ce mode.

Les données obtenues à propos des compétences sur l'emploi et celles sur les pratiques sont assez cohérentes. Il a été effectivement constaté que les apprenants ne sont pas compétents pour saisir les fonctions du subjonctif et ils ne savent pas distinguer les emplois entre le subjonctif et l'indicatif. Il a été 
conclu qu'une grande majorité des étudiants ne peuvent pas employer la fonction volitive du subjonctif bien qu'ils déclarent le volitif turc comme l'équivalent de ce mode. Par ailleurs, très près de la moitié des apprenants n'ont pas réagi vers l'emploi de la possibilité du subjonctif. Quant à la fonction de doute de ce mode, il a été déterminé que les étudiants ont de la difficulté à adopter cette fonction et ils n'arrivent pas à utiliser la fonction déontique de ce mode. Les données obtenues concernant la fonction de l'impératif du subjonctif nous permettent de conclure que $40 \%$ des apprenants ne parviennent pas à employer cette fonction. Il a été précisé que plus de la moitié des étudiants ne peuvent pas employer la fonction opposition de ce mode et la fonction restriction du subjonctif représente une difficulté pour les apprenants. Les données obtenues concernant les emplois de la condition et du but de ce mode nous confirment que les étudiants ne sont pas compétents d'employer ces fonctions. Quant à la fonction incertitude de ce mode, il s'est avéré comme une difficulté importante.

Par conséquent, ces données soutiennent la théorie de Lado, de Fries, d'Odlin, de Wagner selon laquelle "les éléments différents entre les langues rendent l'acquisition de la langue plus difficile et les éléments similaires la rendent facile ». Elles vont par ailleurs dans le même sens que notre hypothèse. En effet, celles-ci démontrent que la difficulté rencontrée par les étudiants universitaires turcs dans l'acquisition du mode subjonctif résulte en grande partie de son caractère de xénité. Les apprenants ont généralement recours au processus de transfert de certaines propriétés grammaticales effectué de leur langue maternelle vers la langue cible tout au long de leur apprentissage. Pourtant, s'agissant du subjonctif, les étudiants turcs n'ont pas cette disposition grammaticale dans leur langue maternelle leur permettant de réaliser ce transfert et qui aurait pu faciliter l'acquisition de ce mode.

En effet, les différences observées sur le plan morphologique et fonctionnel entre le subjonctif français et le volitif turc, paraissent comme l'un des facteurs principaux de la difficulté rencontrée par les apprenants Turcs. Dans ce contexte, il serait intéressant de mener de différentes études contrastives sur les différences linguistiques entre ces deux langues. Il faut par ailleurs prendre en considération les différences entre les langues lors de la préparation des manuels et des méthodes d'enseignement. Compte tenu de ces différences fonctionnelles et structurales, au lieu d'adopter une approche structurale au cours de l'apprentissage mais aussi dans les méthodes, il serait plus profitable de partir de la fonction. Cela pourrait minimiser la difficulté vécue par les apprenants turcs. Une pédagogie comparative du subjonctif peut être plus efficace dans l'acquisition de cette matière.

\section{Bibliographie}

Altındağ, M. (2005). Nitel Araştırma Teknikleri. Yüksek Lisans Tezi. Ankara: Ankara Üniversitesi. 
Topaloğlu, Y. (2017). Acquisition du Subjonctif par Les Étudiants Turcs. Humanitas, 5(10), 333-361

Balcı, A. (2015). Sosyal Bilimlerde Araştırma Yöntem, Teknik ve Illkeler. Ankara: Pegem Akademi.

Bosnalı, S., \& Topaloğlu, Y. (2015). Fransızca Öznellik ve Türkçe Dilek-İstek Kipleri. Uludağ Üniversitesi Eğitim Fakültesi Dergisi, 28(Özel Sayı), 273-284.

Bouton, C. (1984). La linguistique appliquée. Que sais-je? Paris: Presses Universitaire de France.

Březinová, M. (2009). L'interférence linguistique en enseignement de la langue française. Diplomová práce, Pedagogická fakulta Katedra francouzského jazyka a literatury: Masarykova Univerzita.

Cellard, J. (1996). Le subjonctif: Comment l'écrire? - quand l'employer? Belgium: Duculot.

Corder, S. P. (1981). Error Analysis and Interlanguage. London: Oxford University Press.

Delatour, Y., Jennepin, D., Léon-Dufour, M., \& Teyssier, B. (2004). Nouvelle grammaire du Français. Paris: Hachette Livre.

Dubois, J., \& Lagane, R. (2005). Larousse: Livres de bord, grammaire. Montrouge: Baume- Les-Dames.

Fries, C. C. (1945). Teaching \& Learning English as a Foreign Language. USA: The University of Michigan Press.

Gaonac'h, D. (2009). L'apprentissage précoce d'une langue étrangère: Le point de vue de la psycholinguistique. Paris, France: Achevé d'imprimer, Hachette Education.

Grevisse, M., \& Goosse, A. (2011). Le bon usage, grammaire langue française. 15. basim, Paris: De Boeck \& Duculot.

Klein, W. (1989). L'acquisition de langue étrangère. Paris: Athenäum Taschenbücher Linguistik.

Korkmaz, Z. (2009). Türkiye Türkçesi Grameri: Şekil bilgisi. Ankara: Türk Dil Kurumu Yayınları.

-(1992). Gramer Terimleri Sözlüğ̈̈. Ankara: TDK Yayınlar1 575.

Krashen, S. (2013). Second Language Acquisition: Theory, Applications, and Some Conjectures. Cambridge: Cambridge University Press.

-(1981). Second Language Acquisition and Second Language Learning. California: Pergamon Press Inc.

Krashen, S. D. (1982). Principles and Practice in Second Language Acquisition. California: Pergamon Press Inc.

Lado, R. (1957). Linguistics across cultures: Applied linguistics for language teachers. United states of America: The University of Michigan.

Martinez, P. (2008). La didactique des langues etrangères. Que sais-je? Paris: Presses Universitaires de France. 
Topaloğlu, Y. (2017). Acquisition du Subjonctif par Les Étudiants Turcs. Humanitas, 5(10), 333-361

Odlin, T. (1997). Language Transfer: Crosse-linguistique influence in language learning. New York: Cambridge University Press.

Palmer, F. R. (2001). Mood and modality. Cambridge: Cambridge University Press.

Poisson-Quinton, S., Mimran, R., \& Mahéo-Le Coadic, M. (2002). Grammaire expliquée dufrançais. France: CLE International.

Porcher, L. (2003). L'apprentissage précoce des langues. Dominique Groux, Paris: Presses Universitaires De France.

Soutet, O. (2000). Le subjonctif en français. Paris, France: Ophrys.

Trawiński, M. (2005). An Outline of Second Language Acquisition Theories. Kraków: Wydawnictwo Naukowe Akademii.

Wagner, K. H. (1982). Kontrastive Linguistik. D. Aksan içinde, Dilbilim Seçkisi: Günümüz Dílbilimiyle İlgili Yazılardan çeviriler (E. Sözer, Çev., s. 217-224). $\quad$ Ankara: Türk Dil Kurumu Yayınları.

Weinrich, H. (1986). Petite xénologie des langues étrangères. Persee, In: Communications, 43. Le croisement des cultures., 187-203. 12 19, $2014 \quad$ tarihinde

file:///C:/Users/Yusuf\%20Topaloglu/Downloads/article_comm_05888018_1986_num_43_1_1647.pdf adresinden alind1. (2013). La langue? Non: les langues! Trivium. Revue francoallemande de sciences humaines et sociales-Deutsch-französische Zeitschrift für Geistes- und Sozialwissenschaften, 15, 9-11. 1 19, 2015 tarihinde http://trivium.revues.org/4707 adresinden alınd1.

Weinrich, U. (1953). Languages in Contact: Findings and Problems. New York: Publications of the Linguistique Ciecle of New York.

Youssef, N. (2014). Propositions pour l'enseignement du subjonctif aux adultes arabophones: Application aux étudiants des universités syriennes. Pontoise: Thèse de Doctorat en Sciences du Langage Université de Cergy-Pontoise. 06 01, 2015 tarihinde https://tel. archives- ouvertes. fr/tel- 00839941v1 adresinden alındı.

\section{ACQUISITION OF FRENCH SUBJUNCTIVE MODE BY TURKISH UNIVERSITY STUDENTS}

Abstract: The present study handles the subject of the linguistic dimension of the challenges in the context of subjunctive mode encountered by Turkish University students in the process of learning French. Both acquisition and transfer of the mode in question are one of the most important problems encountered. Indeed, we experienced ourselves these difficulties of acquiring French subjunctive mode very closely as being students in the past and educationalist of today. We believe that the main factor underlying this difficulty is the differences between languages. The present study was studied in the frame of applied 
linguistics. Besides, the French subjunctive mode and event modalities suggested to be its equivalent were examined with contrastive approach. The idea that subjunctive mode as morphological and functional is very different for Turkish university students is put forward and the differences caused difficulties in the acquisition process are investigated. This study was evaluated within the framework of the quantitative research method. A questionnaire technique was used in collecting data; quantitative data were obtained and analyzed. The questionnaire consists of 37 questions. According to data obtained, the similarities facilitate the acquisition and the differences make it difficult; the subjunctive mode doesn't carry completely the feature of a foreign element as well as the feature of sameness for Turkish university students.

Keywords: Applied Linguistics, Contrastive Linguistics, Language Acquisition, Language Teaching, Subjective Mode. 